\title{
Interconversion of elasticity measurements between two-dimensional shear wave elastography and transient elastography
}

\author{
Yeun-Yoon Kim¹, Myung-Joon Kim¹, Hyun Joo Shin', Haesung Yoon', Ha Yan Kim², \\ Mi-Jung Lee ${ }^{1}$
}

${ }^{1}$ Department of Radiology and Research Institute of Radiological Science, Severance Children's Hospital, ${ }^{2}$ Biostatistics Collaboration Unit, Yonsei University College of Medicine, Seoul, Korea

\begin{abstract}
Aims: To enable comparison and interconversion of elasticity measurements between two-dimensional (2D) shear wave elastography (SWE) and transient elastography (TE). Materials and methods: Elasticities of three phantoms were measured by 2D SWE (supersonic shear imaging) using four probes and TE using two probes. We performed regression analyses to evaluate correlation between the measurements and phantom elasticities, and make converting equations. In pediatric biliary atresia patients who had stiffness measurements by both 2D SWE and TE within 1-year interval, TE measurements were retrospectively converted into correlating 2D SWE values. We compared the calculated values with 2D SWE measurements by intraclass correlation coefficient. Results: Measurements in phantoms varied according to elastography method and probe selection. However, the measurement by both 2D SWE $\left(R^{2}, 0.974-0.985 ; p<0.001\right)$ and TE $\left(R^{2}, 0.996-0.999 ; p<0.001\right)$ showed significant linear correlation with phantom elasticity in all probe settings. From 67 biliary atresia patients (age, 2 months-20 years), agreements between the measured and calculated values were excellent in all 88 examinations within 1-year interval $(\rho=0.828 ; p<0.001)$ and in 63 examinations within 2-month interval $(\rho=0.863, p<0.001)$. Conclusions: The equations enabled interconversion of elasticity values among different probes of 2D SWE and TE and provided reliable estimation of elasticity values for different probe settings in biliary atresia patients.
\end{abstract}

Keywords: liver fibrosis; transient elastography; shear wave elastography; biliary atresia; comparative study

\section{Introduction}

Biliary atresia is a major cause of chronic liver disease and an important indication for liver transplantation in children [1-3]. Assessment of hepatic fibrosis is important for growing children with biliary atresia in terms of disease status evaluation and liver transplantation planning [4-6]. Ultrasound (US) elastography is highly val-

Received 31.10.2017 Accepted 28.12.2017

Med Ultrason

2018, Vol. 20, No 2, 127-133

Corresponding author: Mi-Jung Lee, $\mathrm{MD}, \mathrm{PhD}$

Department of Radiology and Research

Institute of Radiological Science,

Severance Children's Hospital

Yonsei University College of Medicine,

50-1 Yonsei-ro, Seodaemun-gu, Seoul,

Korea 03722

Phone: 82-2-2228-7400, Fax: 82-2-393-3035

E-mail:mj11213@yuhs.ac ued for its convenience, non-invasiveness, and reproducibility for the measurement of liver stiffness in adults [7], and therefore can be a powerful tool for long-term follow-up evaluation even in pediatric patients. Transient elastography (TE) has been suggested to be useful for assessment of hepatic fibrosis in pediatric patients with chronic liver disease, including biliary atresia [8-11]. Even though there are only a few studies in children, two-dimensional (2D) shear wave elastography (SWE) as well as acoustic radiation force impulse were useful for the differential diagnosis between biliary atresia and other infantile liver diseases [12-14].

Probe selection also should be considered in children. Various probes are needed for liver stiffness measurement (LSM) in children due to their continuous growth and varying body sizes. For TE, the S probe was developed for small children and was previously applied to infants with biliary atresia either before or after Kasai op- 
eration $[11,15]$. For 2D SWE, the small convex probe and high frequency linear probe as well as the large convex probe are available for the evaluation of the liver, which can be especially beneficial to pediatric patients.

The result of LSM in the same individual can be influenced by the probe selection of 2D SWE and TE [1618]. Moreover, the shear wave speed measurement can differ according to elastography system made by different vendors, including TE and 2D SWE [19]. Consequently, appropriate comparison is essential between the results using different systems and probes. In addition, variability of the cut-off values for hepatic fibrosis staging from different systems and probes makes it difficult to directly compare the results of both methods in pediatric patients with chronic liver disease. When using 2D SWE with either SC6-1 or SL15-4 probes for supersonic shear imaging, for example, cut-off values for any fibrosis (F1 or higher) varied from 7.3 to $10.6 \mathrm{kPa}$, while $23.5 \mathrm{kPa}$ was suggested as a cut-off value for liver cirrhosis (F4) $[18,20]$. For TE using either $\mathrm{S}$ or M probes, on the other hand, cut-off value for F1 ranged from 5.1 to $6.1 \mathrm{kPa}$, while that for F4 ranged from 14.1 to 15.15 $\mathrm{kPa}[8,11,21]$. Therefore, the aim of this study was to enable comparison and interconversion of elasticity measurements between 2D SWE and TE with consideration of variable probe settings.

\section{Materials and methods}

We performed a phantom study to establish the relationship between elasticity measurements obtained with 2D SWE and TE which were in the expected range of elasticity values of the patient group. Equations which were made for conversion were retrospectively applied to LSMs of children with biliary atresia for their validation.

\section{Phantom study}

We used commercially available phantom models of uniform elasticity $(3.0,16.9$, and $26.3 \mathrm{kPa})$ for elasticity measurements (The Shear Wave Liver Fibrosis Phantom, model 039, CIRS, Norfolk, VA, USA). 2D SWE (Aixplorer, SuperSonic Imagine, Aix-en-Provence, France) and TE (FibroScan, Echosens, Paris, France) were performed in each phantom to measure the elasticity. We put the phantoms in lateral decubitus position and placed probes perpendicularly to the phantom surface in order to simulate intercostal measurement in the real patient.

The following four probes were used for 2D SWE: SuperCurved probe with 1-6 MHz (SC6-1), SuperLinear probe with 2-10 MHz (SL10-2), SuperMicroConvex probe with 3-12 MHz (SC12-3), and SuperLinear probe with 4-15 MHz (SL15-4). Routine pediatric abdominal setting was used for the SC6-1, SL10-2, and SC12-3 probes, and the setting for superficial lesion evaluation was used for the SL15-4 probe. Circular ROIs were placed in the homogeneous areas of the phantoms. The diameter of the ROI was $6 \mathrm{~mm}$ except for the SC6-1 probe, for which a ROI diameter of $8 \mathrm{~mm}$ was used according to the routine pediatric abdominal setting. The acquisition depth was $4 \mathrm{~cm}$ for the SC6-1 probe, $2 \mathrm{~cm}$ for the SL10-2 and SC12-3 probes, and $1.5 \mathrm{~cm}$ for the SL15-4 probe, according to the routine setting for each probe. Ten valid measurements from separate and repetitive acquisitions were obtained by each probe in the same area of the phantom by a board-certified radiologist. The elasticity measurements were presented as Young modulus in the unit of $\mathrm{kPa}$.

For TE, the S2 probe with the diameter of $5 \mathrm{~mm}$ and the $\mathrm{M}$ probe with the diameter of $7 \mathrm{~mm}$ were used. The ultrasonic frequencies were $5 \mathrm{MHz}$ for the $\mathrm{S} 2$ probe and 3.5 MHz for the M probe. TE was performed by a trained investigator who was certified by the manufacturer. The median value of ten repetitive and valid measurements was obtained with each probe in each phantom. The TE results were also obtained in the unit of $\mathrm{kPa}$.

\section{Examinations in children with biliary atresia}

This retrospective analysis on the patient data was performed with the approval of our Institutional Review Board, and the need for informed consent was waived. All children with biliary atresia who had previously underwent a Kasai operation, and had both results of LSM by 2D SWE and TE performed from January 2015 to July 2016 were included as subjects. The time interval between 2D SWE and TE examinations was limited to one year. We reviewed US images and LSM data obtained by 2D SWE and TE. We also reviewed laboratory results regarding liver function and cholestasis. Signs of portal hypertension, such as splenomegaly, ascites or varices, were collected based on the US, clinical or endoscopic results. Body mass index (BMI) at the time of 2D SWE was defined as normal for percentiles lower than 85, overweight for percentiles between 85 and 95 , and obese for percentiles of 95 or higher $[22,23]$.

2D SWE was performed with either the SC6-1 or SL10-2 probe through an intercostal space with patients in the supine position and regular free breathing. We used the same routine pediatric abdominal setting as in the phantom study. LSMs were obtained three times in the homogeneous liver parenchyma avoiding vessels and bile ducts, and the mean value of the three elasticity measurements was calculated as the representative value [24]. TE was performed with either the S2 or M probe via the intercostal approach. S2 probe was used for children with the thoracic perimeter of $75 \mathrm{~cm}$ or less, and $\mathrm{M}$ probe was used for children with the thoracic perim- 
eter of more than $75 \mathrm{~cm}$. The median value of ten valid measurements was considered to be the representative value. Both methods were conducted in children below three years of age after fasting for four hours and for six hours in older children.

\section{Data and statistical analysis}

Linear regression analyses were performed to evaluate the correlation between the results of $2 \mathrm{D} \mathrm{SWE}$ or TE and phantom elasticity for each probe setting. Because unexpectedly we could not obtain elasticity values using either $\mathrm{S} 2$ or $\mathrm{M}$ probe in the $3.0 \mathrm{kPa}$ phantom, ten values were imputed for missing values in each probe setting by a Markov Chain Monte Carlo method. Linear regression analysis was performed for the data with or without imputed values in TE measurements. Using the equations derived from the linear correlation analyses, the corresponding elasticity values in 2D SWE with each probe were calculated from the elasticity values in TE with the $\mathrm{S} 2$ and $\mathrm{M}$ probe, respectively.

For the patient data, we also calculated the 2D SWE values corresponding to the LSMs by TE using the equations. We then analyzed the agreement between the LSM by 2D SWE and the statistically estimated values for 2D SWE using intraclass correlation coefficient for average measures. Analyses were done both for all examinations (with less than 1-year interval) and for those with less than 2-month interval between 2D SWE and TE. We also analyzed the results in each BMI group.

Statistical Analysis Software (SAS) version 9.2 (SAS Institute Inc., Cary, NC, USA) and SPSS Statistics version 20.0 (IBM Corporation, Armonk, NY, USA) were used for the analysis. A $p$ value less than 0.05 was considered to indicate statistical significance.

\section{Results}

\section{Equations from the phantom study}

The results of elasticity measurements by each probe of $2 \mathrm{D} \mathrm{SWE}$ in $3.0,16.9$, and $26.3 \mathrm{kPa}$ phantoms tended to have larger discrepancies in the stiffer phantoms and to be relatively lower than the actual phantom elasticities. However, the results of 2D SWE with all four probes showed significant linear correlation with phantom elasticity $\left(\mathrm{R}^{2}, 0.974-0.985 ; \mathrm{p}<0.001\right)$. The following equations demonstrate the relationships between the phantom elasticity and the results of 2D SWE with each probe.

SC6-1: phantom elasticity $=1.388+(1.314 \times$ the result of 2D SWE) $\left(\mathrm{R}^{2}=0.984\right)$

SL10-2: phantom elasticity $=1.720+(1.410 \times$ the result of $2 \mathrm{D}$ SWE) $\left(\mathrm{R}^{2}=0.985\right)$

$\mathrm{SC12-3}$ : phantom elasticity $=1.083+(1.546 \times$ the result of $2 \mathrm{D} \mathrm{SWE})\left(\mathrm{R}^{2}=0.980\right)$

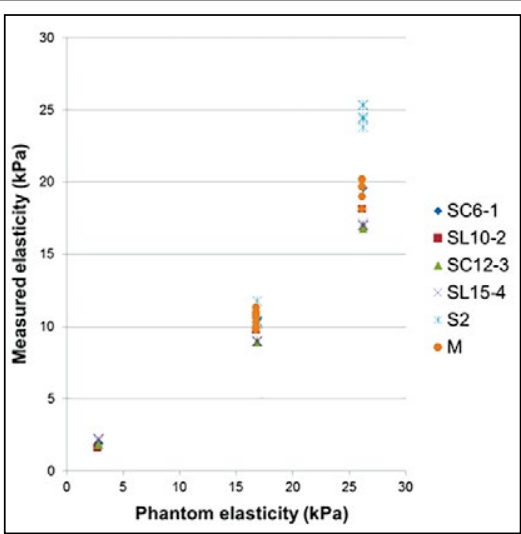

Fig 1. A scatter plot of the elasticity values measured by twodimensional (2D) shear wave elastography (SWE) using four probes (SC6-1, SL10-2, SC12-3, and SL15-4) and transient elastography (TE) using two probes ( $\mathrm{S} 2$ and $\mathrm{M}$ ) in elasticity phantom models of 3.0, 16.9, and $26.3 \mathrm{kPa}$. SC6-1 = SuperCurved probe with 1-6 MHz, SL10-2 = SuperLinear probe with 2-10 MHz, SC12-3 = SuperMicroConvex probe with 3-12 $\mathrm{MHz}, \mathrm{SL15-4}=$ SuperLinear probe with 4-15 MHz.

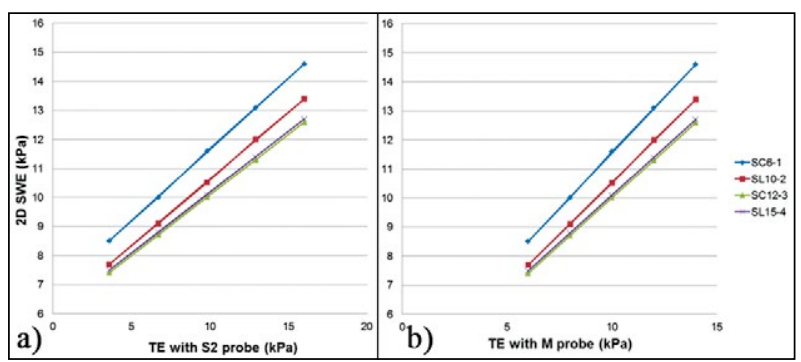

Fig 2. Linear correlations between representative elasticity values in TE and the corresponding elasticity values in 2D SWE with each probe based on the equations from the phantom study: a) Correlations between TE with the $\mathrm{S} 2$ probe and 2D SWE with each probe; b) Correlations between TE with the M probe and 2D SWE with each probe.

SL15-4: phantom elasticity $=0.943+(1.549 \times$ the result of $2 \mathrm{D} \mathrm{SWE})\left(\mathrm{R}^{2}=0.974\right)$

In $\mathrm{TE}$, the elasticity values for $\mathrm{M}$ probe tended to be lower than those for S2 probe for the same correlating phantom elasticity (fig 1). When excluding the imputed values for the $3.0 \mathrm{kPa}$ phantom setting, elasticity values of both the S2 probe (regression coefficient 0.655, $\mathrm{R}^{2}=0.996, \mathrm{p}<0.001$ ) and $\mathrm{M}$ probe (regression coefficient $1.011, \mathrm{R}^{2}=0.984, \mathrm{p}<0.001$ ) demonstrated significant linear correlation with phantom elasticity. When including the imputed values, the correlation was also significant for elasticity values of both the $\mathrm{S} 2$ and $\mathrm{M}$ probes $\left(\mathrm{R}^{2}\right.$, 0.996-0.999; $\mathrm{p}<0.001)$ and the relationships are demonstrated in the following equations.

$\mathrm{S} 2$ : phantom elasticity $=10.138+(0.655 \times$ the result of TE) $\left(\mathrm{R}^{2}=0.999\right)$ 
M: phantom elasticity $=6.445+(1.102 \times$ the result of TE) $\left(\mathrm{R}^{2}=0.996\right)$

The difference between measurements by the $\mathrm{S}$ probe and $\mathrm{M}$ probe also showed a tendency to be larger in the stiffer phantoms and greater in the $26.3 \mathrm{kPa}$ phantom than the differences among measurements by 2D SWE probes. Different linear correlations are demonstrated in fig 2 by correlating representative elasticity values in TE with the S2 probe (fig $2 a$ ) or M probe (fig $2 b$ ) to the corresponding elasticity values in $2 \mathrm{D}$ SWE with each probe using the aforementioned equations. Representative values in TE with the S2 probe (3.6, 6.7, 9.8, 12.9, and 16 $\mathrm{kPa})$ were calculated from arbitrary representative values in TE with the M probe $(6,8,10,12$, and $14 \mathrm{kPa}$, respectively) based on the equations (Table I).

Application of the equations in children with biliary atresia

During the study period, 67 children (27 boys and 40 girls) underwent 88 abdominal US examinations including 2D SWE and TE within a 1-year interval. The mean time interval between 2D SWE and TE was 62.4 days, and 63 of 88 examinations were performed within a 2 -month interval. The age range of patients was 2 months to 20 years, with a mean of $6.6 \pm 4.5$ years. The majority ( 80 of $88,90.9 \%$ ) of examinations was performed in patients with normal BMI, except for four overweight and

Table I. Equations and representative values of phantom elasticity and elasticity measurements

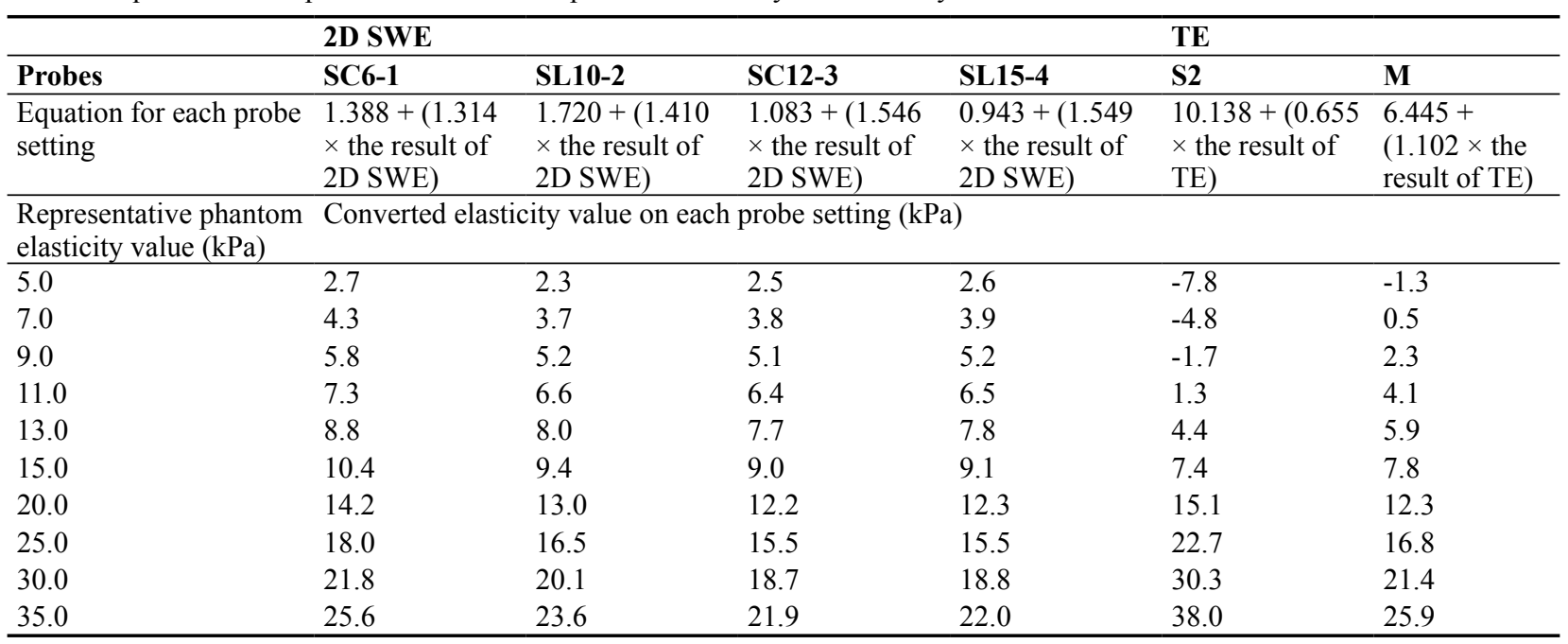

2D SWE - 2 dimensional shear wave elastography; TE - transient elastography

Table II. Characteristics of 67 biliary atresia patients

\begin{tabular}{ll}
\hline Characteristics & Mean \pm standard deviations \\
\hline M:F & $27: 40$ \\
Age at Kasai operation (days) & $61.3 \pm 28.1$ \\
Age at examination (years) & $6.6 \pm 4.5$ \\
Body weight $(\mathrm{kg})$ & $26.0 \pm 15.3$ \\
Body mass index $\left(\mathrm{kg} / \mathrm{m}^{2}\right)$ & $17.2 \pm 2.7$ \\
Laboratory findings at the time of 2D SWE & \\
Total bilirubin (mg/dL) & $1.4 \pm 2.1$ \\
Direct bilirubin $(\mathrm{mg} / \mathrm{dL})$ & $0.8 \pm 1.7$ \\
Albumin $(\mathrm{g} / \mathrm{dL})$ & $4.0 \pm 0.5$ \\
AST (IU/L) & $76.8 \pm 83.7$ \\
ALT (IU/L) & $63.1 \pm 70.0$ \\
ALP (IU/L) & $309.1 \pm 153.6$ \\
GGT (IU/L) & $121.3 \pm 163.4$ \\
PT-INR & $1.12 \pm 0.35$ \\
Platelet count $\left(10^{9} / \mathrm{L}\right)$ & $165.3 \pm 88.2$ \\
Presence of portal hypertension sign (n, \%) & $43(64.2 \%)$ \\
\hline AST aspatate aming
\end{tabular}

AST - aspartate aminotransferase; ALT - alanine aminotransferase; ALP - alkaline phosphatase; GGT - gamma glutamyl transpeptidase; PT-INR - prothrombin time-international normalized ratio 
four obese patients. The laboratory data for liver function and cholestasis were mostly stable at the time of examination (Table II). Forty-three (64.2\%) of 67 patients had the sign of portal hypertension based on the US, clinical and endoscopic examinations.

For 2D SWE, the SC6-1 probe and SL10-2 probe were used in 72 and 16 examinations, respectively. The LSM ranges of 2D SWE were 4.6 to $35.7 \mathrm{kPa}$ with a mean of $12.1 \pm 6.5 \mathrm{kPa}$ in examinations by SC6-1 probe and 6.1 to $29.5 \mathrm{kPa}$ with a mean of $14.9 \pm 6.6 \mathrm{kPa}$ in examinations by SL10-2 probe. For TE, the S2 probe and $\mathrm{M}$ probe were used in 77 and 11 examinations, respectively. The LSM ranges of TE were 3.9 to $75.0 \mathrm{kPa}$ with a mean of $15.8 \pm 13.0 \mathrm{kPa}$ in examinations by $\mathrm{S} 2$ probe and 6.6 to $24.3 \mathrm{kPa}$ with a mean of $11.5 \pm 5.1 \mathrm{kPa}$ in examinations by $\mathrm{M}$ probe. With respect to the probe selection in 2D SWE and TE, there were 62 examinations by SC6-1 probe and $\mathrm{S} 2$ probe, 15 examinations by SL10-2 probe and S2 probe, 10 examinations by SC6-1 probe and $\mathrm{M}$ probe, and one examination by SL10-2 probe and M probe.

2D SWE values estimated by our equations from the LSM with TE had excellent agreements with the LSM by 2D SWE both in all 88 examinations within a 1 -year interval $(\rho=0.828, \mathrm{p}<0.001)$ and in 63 examinations within a 2-month interval $(\rho=0.863, p<0.001)$ between 2D SWE and TE (fig 3a). When considering patients' BMI, 80 examinations of children with normal BMI showed an excellent agreement between the estimated and measured values of $2 \mathrm{D} \operatorname{SWE}(\rho=0.832, \mathrm{p}<0.001)$. The measurements in overweight or obese patients $(n=8)$ also had the tendency of agreement with the estimated values of LSM (fig 3b).

\section{Discussions}

To the best of our knowledge, this is the first study suggesting equations for converting LSMs by each probe of 2D SWE and TE to the correlating elasticity values in various probe settings. Our results demonstrate that elasticity values differ according to the elastographic methods and probes. There was a tendency of larger discrepancies among the elasticity values in the stiffer phantoms, especially between the results of S probe and $\mathrm{M}$ probe in the $26.3 \mathrm{kPa}$ phantom. In TE, the elasticity values for $\mathrm{M}$ probe tended to be lower than those for $\mathrm{S} 2$ probe for the same correlating phantom elasticity, which was in accordance with previous results $[9,15,25]$. In 2D SWE, stiffness values for SC6-1 were higher than those for SL15-4 when correlated with our equations, as with the result of the previous study on the pediatric population [18]. Another phantom study has also shown that SWV measurements by SC6-1 probe had a tendency to

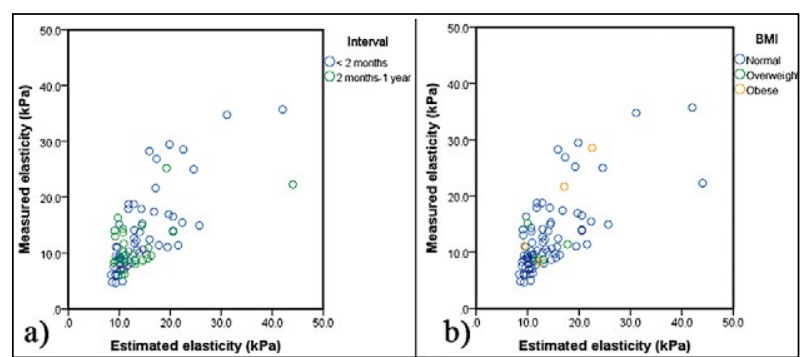

Fig 3. Scatter plots of measured and estimated 2D SWE values in children with biliary atresia: a) scatter plot of 2D SWE measurements and statistically estimated 2D SWE values from the TE measurements shows excellent agreement, not only in 63 examinations $(\rho=0.863, p<0.001)$ with less than a 2 -month interval (blue circles) but also in all data $(\rho=0.828$; $p<0.001)$ including the other 25 examinations with the interval of two months to one year (green circles); b) scatter plot of 2D SWE measurements and statistically estimated 2D SWE values from TE measurements according to the body mass index (BMI). Eighty examinations of children with normal BMI $(<85$ percentiles, blue circles) show excellent agreement $(\rho=0.832$, $\mathrm{p}<0.001$ ). Four examinations of overweight children (BMI: 8595 percentiles, green circles) and the other four examinations of obese children (BMI $>95$ percentiles, orange circles) also show the tendency of agreement.

be higher than those by the SL10-2 probe [26]. We assume that technical differences such as probe frequency, and vibration amplitude may contribute to the discrepancy in values among the probes.

When the equations were applied to the patient data, reliable estimation and conversion of elasticity values were feasible. The 2D SWE values estimated by our equations from TE measurements correlated well with the 2D SWE measurements. In addition, high coefficients were attained both for patients with short and long intervals between 2D SWE and TE studies. As most patients had stable liver function and cholestatic level, there may have been small differences in the disease status between examinations with long intervals. The good correlations were also consistently demonstrated in the normal, overweight and obese patients, which suggest the applicability of our equations to patients with varied BMIs.

In the phantom study, TE failed to produce any valid measurement in the $3.0 \mathrm{kPa}$ phantom, while 2D SWE successfully obtained elasticity measurements in all phantoms. The measurement failure of TE in the $3.0 \mathrm{kPa}$ phantom could be attributed to relatively low stiffness of the phantom which might have generated signal error or loss. However, measurement value as low as $1.6 \mathrm{kPa}$ has been successfully obtained by TE on polyvinyl alcohol cryogel phantom in a previous study [27]. Although we could not compare the failure rates between 2D SWE and TE in the patient data due to retrospective design, higher failure rate of TE than 2D SWE has been demonstrated 
both in pediatric and adult populations in previous studies [28-30]. Meanwhile, a study with a high prevalence of obesity showed a higher failure rate of 2D SWE compared to TE [31]. Achieving reliable measurements also could be affected by the operator experience and patient body habitus [32].

This study has some limitations. First, we did not correlate the elastographic results with the pathologic results. Liver biopsy was not included in the routine management plan for pediatric patients in our institution and was not performed due to its invasiveness and the retrospective nature of our analysis. Future studies which control patient factors influencing the LSM value, including cholestasis, and use liver biopsy result as a reference standard are warranted to further validate our results. Secondly, we could not apply our equations to the measurements by SC12-3 and SL15-4 probes in the patient data, as these probes were not used in the included patients. However, our study demonstrated excellent agreements using the equations in various probe combinations of 2D SWE (SC6-1 and SL10-2 probes) and TE (S2 and M probes). Further studies may expand the usage of our equations in other probe combinations. Thirdly, the time interval of 1 year between 2D SWE and TE was relatively wide. Therefore, we separately performed analysis for examinations of a time interval within 2 months and found that examinations having a 2-month interval as well as a 1-year interval had good correlation results. Lastly, we only used the mean or median values for elasticity measurements but did not analyze technical quality parameters. Not only obtaining and comparing values, but also evaluating value reliability is important in quantitative analyses. Additional research is required for this point.

\section{Conclusions}

There were significant linear correlations between elasticity measurements by 2D SWE and TE in the phantom study. The proposed equations enabled the interconversion of elasticity values among different probes of 2D SWE and TE and provided reliable estimation of hepatic elasticity values for different probe settings in biliary atresia patients.

\section{Conflict of interest: none}

\section{References}

1. Chardot C, Carton M, Spire-Bendelac N, Le Pommelet C, Golmard JL, Auvert B. Prognosis of biliary atresia in the era of liver transplantation: French national study from 1986 to 1996 . Hepatology 1999;30:606-611.
2. Haafiz AB. Liver fibrosis in biliary atresia. Expert Rev Gastroenterol Hepatol 2010;4:335-343.

3. Hartley JL, Davenport M, Kelly DA. Biliary atresia. Lancet 2009;374:1704-1713.

4. Hahn SM, Kim S, Park KI, Han SJ, Koh H. Clinical benefit of liver stiffness measurement at 3 months after Kasai hepatoportoenterostomy to predict the liver related events in biliary atresia. PLoS One 2013;8:e80652.

5. Chongsrisawat V, Vejapipat P, Siripon N, Poovorawan Y. Transient elastography for predicting esophageal/gastric varices in children with biliary atresia. BMC Gastroenterol 2011;11:41.

6. Hanquinet S, Courvoisier DS, Rougemont AL, et al. Acoustic radiation force impulse sonography in assessing children with biliary atresia for liver transplantation. Pediatr Radiol 2016;46:1011-1016.

7. Jeong WK, Lim HK, Lee HK, Jo JM, Kim Y. Principles and clinical application of ultrasound elastography for diffuse liver disease. Ultrasonography 2014;33:149-160.

8. Fitzpatrick E, Quaglia A, Vimalesvaran S, Basso MS, Dhawan A. Transient elastography is a useful noninvasive tool for the evaluation of fibrosis in paediatric chronic liver disease. J Pediatr Gastroenterol Nutr 2013;56:72-76.

9. Goldschmidt I, Streckenbach C, Dingemann C, et al. Application and limitations of transient liver elastography in children. J Pediatr Gastroenterol Nutr 2013;57:109-113.

10. de Ledinghen V, Le Bail B, Rebouissoux L, et al. Liver stiffness measurement in children using FibroScan: feasibility study and comparison with Fibrotest, aspartate transaminase to platelets ratio index, and liver biopsy. J Pediatr Gastroenterol Nutr 2007;45:443-450.

11. Shen QL, Chen YJ, Wang ZM, et al. Assessment of liver fibrosis by Fibroscan as compared to liver biopsy in biliary atresia. World J Gastroenterol 2015;21:6931-6936.

12. Leschied JR, Dillman JR, Bilhartz J, Heider A, Smith EA, Lopez MJ. Shear wave elastography helps differentiate biliary atresia from other neonatal/infantile liver diseases. Pediatr Radiol 2015;45:366-375.

13. Wang X, Qian L, Jia L, et al. Utility of Shear Wave Elastography for Differentiating Biliary Atresia From Infantile Hepatitis Syndrome. J Ultrasound Med 2016;35:14751479.

14. Hanquinet S, Courvoisier DS, Rougemont AL, et al. Contribution of acoustic radiation force impulse (ARFI) elastography to the ultrasound diagnosis of biliary atresia. Pediatr Radiol 2015;45:1489-1495.

15. Kim S, Kang Y, Lee MJ, Kim MJ, Han SJ, Koh H. Points to be considered when applying FibroScan $\mathrm{S}$ probe in children with biliary atresia. J Pediatr Gastroenterol Nutr 2014;59:624-628.

16. Myers RP, Pomier-Layrargues G, Kirsch R, et al. Feasibility and diagnostic performance of the FibroScan XL probe for liver stiffness measurement in overweight and obese patients. Hepatology 2012;55:199-208.

17. Ferraioli G, Lissandrin R, Zicchetti M, Filice C. Assessment of liver stiffness with transient elastography by using $\mathrm{S}$ and M probes in healthy children. Eur J Pediatr 2012;171:1415. 
18. Franchi-Abella S, Corno L, Gonzales E, et al. Feasibility and Diagnostic Accuracy of Supersonic Shear-Wave Elastography for the Assessment of Liver Stiffness and Liver Fibrosis in Children: A Pilot Study of 96 Patients. Radiology 2016;278:554-562.

19. Hall TJ, Milkowski A, Garra B, et al. RSNA/QIBA: Shear wave speed as a biomarker for liver fibrosis staging. Ultrasonics Symposium (IUS) 2013:397-400.

20. Tutar O, Beser OF, Adaletli I, et al. Shear wave elastography in the evaluation of liver fibrosis in children. J Pediatr Gastroenterol Nutr 2014;58:750-755.

21. Nobili V, Vizzutti F, Arena U, et al. Accuracy and reproducibility of transient elastography for the diagnosis of fibrosis in pediatric nonalcoholic steatohepatitis. Hepatology 2008;48:442-448.

22. Cole TJ, Bellizzi MC, Flegal KM, Dietz WH. Establishing a standard definition for child overweight and obesity worldwide: international survey. BMJ 2000;320:1240-1243.

23. Park HW, Yoo HY, Kim CH, et al. Reference values of body composition indices: the Korean National Health and Nutrition Examination Surveys. Yonsei Med J 2015;56:95-102.

24. Shin HJ, Kim MJ, Kim HY, Roh YH, Lee MJ. Optimal Acquisition Number for Hepatic Shear Wave Velocity Measurements in Children. Plos One 2016;11:e0168758.

25. Pradhan F, Ladak F, Tracey J, Crotty P, Myers RP. Feasibility and reliability of the FibroScan S2 (pediatric) probe compared with the $\mathrm{M}$ probe for liver stiffness measurement in small adults with chronic liver disease. Ann Hepatol 2013;12:100-107.
26. Shin HJ, Kim MJ, Kim HY, Roh YH, Lee MJ. Comparison of shear wave velocities on ultrasound elastography between different machines, transducers, and acquisition depths: a phantom study. Eur Radiol 2016;26:3361-3367.

27. Cournane S, Cannon L, Browne JE, Fagan AJ. Assessment of the accuracy of an ultrasound elastography liver scanning system using a PVA-cryogel phantom with optimal acoustic and mechanical properties. Phys Med Biol 2010;55:5965-5983.

28. Leung VY, Shen J, Wong VW, et al. Quantitative elastography of liver fibrosis and spleen stiffness in chronic hepatitis B carriers: comparison of shear-wave elastography and transient elastography with liver biopsy correlation. Radiology 2013;269:910-918.

29. Bota S, Paternostro R, Etschmaier A, et al. Performance of 2-D Shear Wave Elastography in Liver Fibrosis Assessment Compared with Serologic Tests and Transient Elastography in Clinical Routine. Ultrasound Med Biol 2015;41:2340-2349.

30. Engelmann G, Gebhardt C, Wenning D, et al. Feasibility study and control values of transient elastography in healthy children. Eur J Pediatr 2012;171:353-360.

31. Cassinotto C, Lapuyade B, Mouries A, et al. Non-invasive assessment of liver fibrosis with impulse elastography: comparison of Supersonic Shear Imaging with ARFI and FibroScan(R). J Hepatol 2014;61:550-557.

32. Gradinaru-Tascau O, Sporea I, Bota S, et al. Does experience play a role in the ability to perform liver stiffness measurements by means of supersonic shear imaging (SSI)? Med Ultrason 2013;15:180-183. 